\title{
Arc Complexes, Sphere Complexes, and Goeritz Groups
}

\author{
Sangbum Cho, Yuya Koda, \& Arim Seo
}

\begin{abstract}
AвSTRACT. We show that if a Heegaard splitting is obtained by gluing a splitting of Hempel distance at least 4 and the genus- 1 splitting of $S^{2} \times S^{1}$, then the Goeritz group of the splitting is finitely generated. To show this, we first provide a sufficient condition for a full subcomplex of the arc complex for a compact orientable surface to be contractible, which generalizes the result by Hatcher that the arc complexes are contractible. We then construct infinitely many Heegaard splittings, including the above-mentioned Heegaard splitting, for which suitably defined complexes of Haken spheres are contractible.
\end{abstract}

\section{Introduction}

Let $\Sigma_{g, n}$ be a compact connected orientable surface of genus $g$ with $n$ holes, where $n \geq 3$ if $g=0$ and $n \geq 1$ if $g \geq 1$. As an analogue of the curve complex, the arc complex $\mathcal{A}_{g, n}$ of $\Sigma_{g, n}$ is defined to be the simplicial complex whose vertices are isotopy classes of essential arcs in $\Sigma_{g, n}$ and whose $k$-simplices are collections of $k+1$ vertices represented by pairwise disjoint and nonisotopic arcs in $\Sigma_{g, n}$. Hatcher [13] proved that the complex $\mathcal{A}_{g, n}$ is contractible. See also Cho, McCullough, and Seo [8], Irmak and McCarthy [15] and Korkmaz and Papadopoulos [18] for related works on arc complexes.

In Section 1, we provide a useful sufficient condition for a full subcomplex of the arc complex to be contractible (Theorem 1.3). Since the arc complex $\mathcal{A}_{g, n}$ itself satisfies this condition, it is contractible, which gives an updated proof for Hatcher's result. Moreover, we also show that the full subcomplex $\mathcal{A}_{g, n}^{*}$ of $\mathcal{A}_{g, n}$, with $n \geq 2$, spanned by vertices of arcs connecting different boundary components is contractible.

A genus- $g$ Heegaard splitting of a closed orientable 3-manifold $M$ is a decomposition of the manifold into two handlebodies of the same genus $g$. That is, $M=V \cup W$ and $V \cap W=\partial V=\partial W=\Sigma$, where $V$ and $W$ are handlebodies of genus $g$, and $\Sigma$ is their common boundary surface. We simply denote by $(V, W ; \Sigma)$ the splitting and call the surface $\Sigma$ the Heegaard surface of the splitting. It is well known that every closed orientable 3-manifold admits a genus- $g$ Heegaard splitting for some genus $g \geq 0$. Given a genus- $g$ Heegaard

Received February 27, 2015. Revision received August 24, 2015.

The first-named author is supported by Basic Science Research Program through the National Research Foundation of Korea(NRF) funded by the Ministry of Science, ICT and Future Planning (NRF-2015R1A1A1A05001071). The second-named author is supported by JSPS Postdoctoral Fellowships for Research Abroad, and by the Grant-in-Aid for Young Scientists (B), JSPS KAKENHI Grant Number 26800028. 
splitting $(V, W ; \Sigma)$ with $g \geq 2$ for $M$, a separating sphere $P$ embedded in $M$ is called a Haken sphere if $P \cap \Sigma$ is a single essential simple closed curve in $\Sigma$. Two Haken spheres $P$ and $Q$ are said to be equivalent if $P \cap \Sigma$ is isotopic to $Q \cap \Sigma$ in $\Sigma$. When the splitting $(V, W ; \Sigma)$ admits Haken spheres, we denote by $\mu=\mu(V, W ; \Sigma)$ the minimal cardinality of $P \cap Q \cap \Sigma$, where $P$ and $Q$ vary over all pairwise nonequivalent Haken spheres for the splitting. The sphere complex for the splitting $(V, W ; \Sigma)$ is then defined to be the simplicial complex whose vertices are equivalence classes of Haken spheres for the splitting and whose $k$-simplices are collections of $k+1$ vertices represented by Haken spheres $P_{0}, P_{1}, \ldots, P_{k}$, respectively, such that the cardinality of $P_{i} \cap P_{j} \cap \Sigma$ is $\mu$ for all $0 \leq i<j \leq k$.

The structures of sphere complexes for genus-2 Heegaard splittings have been studied by several authors. If a genus-2 Heegaard splitting for a 3-manifold admits Haken spheres, then the manifold is one of $S^{3}, S^{2} \times S^{1}$, lens spaces, and their connected sums. It is known that the sphere complex for the genus-2 Heegaard splitting of $S^{3}$ is connected and even contractible from Scharlemann [24], Akbas [1], and Cho [3]. Lei [19] and Lei and Zhang [20] proved that the sphere complexes are connected for genus-2 Heegaard splittings of nonprime 3-manifolds, that is, the connected sum whose summands are lens spaces or $S^{2} \times S^{1}$, and later, Cho and Koda [7] showed that they are actually contractible.

In Section 2, we study the Heegaard splitting for a 3-manifold having a single $S^{2} \times S^{1}$ summand in its prime decomposition. We prove that if a genus- $g$ Heegaard splitting with $g \geq 2$ is the splitting obtained by gluing a genus- $(g-1)$ Heegaard splitting of Hempel distance at least 2 and the genus-1 Heegaard splitting of $S^{2} \times S^{1}$, then its sphere complex is a contractible, $(4 g-5)$-dimensional complex (Corollary 2.7). In fact, we show that the sphere complex is isomorphic to the full subcomplex $\mathcal{A}_{g-1,2}^{*}$ of the arc complex $\mathcal{A}_{g-1,2}$. As a special case, the sphere complex for the genus-2 Heegaard splitting of $S^{2} \times S^{1}$ is a contractible, three-dimensional complex (Corollary 2.8).

For a Heegaard splitting $(V, W ; \Sigma)$ for a 3-manifold, the Goeritz group is defined to be the group of isotopy classes of the orientation-preserving homeomorphisms of the manifold that preserve $V$ and $W$ setwise. We might expect that the Goeritz group would be simpler once we have more complicated Heegaard splitting in some sense. One of the important results on Goeritz group in this view point is that the Goeritz groups of Heegaard splittings of Hempel distance at least 4 are all finite groups, which is given in Johnson [16]. On the other hand, it is hard to determine whether the Goeritz group of a given Heegaard splitting of low Hempel distance is finitely generated or not. Even it remains open whether the Goeritz group of a Heegaard splitting of genus at least 3 for the 3 -sphere is finitely generated or not. The Goeritz groups of genus-1 Heegaard splittings are easy to describe, and for genus-2 reducible Heegaard splittings, their Goeritz groups have been studied in $[10 ; 24 ; 1 ; 3 ; 4 ; 5 ; 6 ; 7]$.

In the final section, we study the Goeritz groups of the Heegaard splittings given in Section 2. The main result is that, for a Heegaard splitting obtained by 


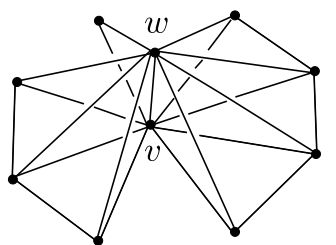

(a)

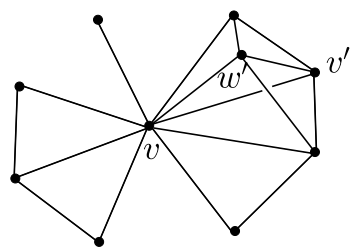

(b)

Figure 1

gluing a Heegaard splitting of Hempel distance at least 4 and the genus-1 Heegaard splitting of $S^{2} \times S^{1}$, its Goeritz group is finitely generated (Corollary 3.4). This can be compared with the result of Johnson [17], who showed that if a Heegaard splitting is obtained by gluing a Heegaard splitting of high Hempel distance and the genus- 1 Heegaard splitting of $S^{3}$, then its Goeritz group is finitely generated.

Throughout the paper, we will work in the PL category. By $\operatorname{Nbd}(X ; Y)$ we denote a regular neighborhood of a subspace $X$ of a polyhedral space $Y$.

\section{Arc Complexes}

We start with recalling a sufficient condition for contractibility of a simplicial complex, introduced in [3], which is a generalization of the proof of Theorem 5.3 in [21].

Let $\mathcal{K}$ be a simplicial complex. We say that a vertex $w$ is adjacent to a vertex $v$ of $\mathcal{K}$ if $w$ equals $v$ or if $w$ is joined to $v$ by an edge of $\mathcal{K}$. We denote by $\operatorname{st}(v)$ the star of a vertex $v$ of $\mathcal{K}$ that is the full subcomplex of $\mathcal{K}$ spanned by the vertices adjacent to $v$. An adjacency pair $(X, v)$ in $\mathcal{K}$ is a finite multiset that consists of vertices of $s t(v)$. Here the finite multiset $X$ is a finite set $\left\{v_{1}, v_{2}, \ldots, v_{k}\right\}$ allowed to have $v_{i}=v_{j}$ for some $1 \leq i<j \leq k$. A remoteness function for a vertex $v_{0}$ of $\mathcal{K}$ is a function $r$ from the set of vertices of $\mathcal{K}$ to $\mathbb{N} \cup\{0\}$ satisfying $r^{-1}(0) \subset$ $s t\left(v_{0}\right)$. A blocking function for a remoteness function $r$ is a function $b$ from the set of adjacency pairs of $\mathcal{K}$ to $\mathbb{N} \cup\{0\}$ satisfying the following properties for every adjacency pair $(X, v)$ with $r(v)>0$ :

(1) If $b(X, v)=0$, then there exists a vertex $w$ of $s t(v)$ such that $r(w)<r(v)$ and $(X, w)$ is also an adjacency pair (see Figure $1(\mathrm{a}))$.

(2) If $b(X, v)>0$, then there exist an element $v^{\prime}$ of $X$ and a vertex $w^{\prime}$ of $s t\left(v^{\prime}\right)$ such that

(a) $r\left(w^{\prime}\right)<r\left(v^{\prime}\right)$

(b) if an element $x$ of $X$ is adjacent to $v^{\prime}$, then $x$ is also adjacent to $w^{\prime}$, and

(c) $b\left(X \backslash\left\{v^{\prime}\right\} \cup\left\{w^{\prime}\right\}, v\right)<b(X, v)$, where $X \backslash\left\{v^{\prime}\right\} \cup\left\{w^{\prime}\right\}$ is the multiset obtained by removing one instance of $v^{\prime}$ from $X$ and adding one instance of $w^{\prime}$ to $X$ (see Figure 1(b)). 
A simplicial complex $\mathcal{K}$ is called a flag complex if any collection of pairwise distinct $k+1$ vertices span a $k$-simplex whenever any two of them span a 1 simplex.

Lemma 1.1 ([3], Prop. 3.1). Let $\mathcal{K}$ be a flag complex with a base vertex $v_{0}$. If there exists a remoteness function $r$ on the set of vertices of $\mathcal{K}$ for $v_{0}$ that admits a blocking function $b$, then $\mathcal{K}$ is contractible.

The idea of the proof given in [3] is to show that the homotopy groups are all trivial. That is, given any simplicial map $f: S^{q} \rightarrow \mathcal{K}, q \geq 0$, with respect to a triangulation $\Delta$ of $S^{q}$, we find a simplicial map $g: S^{q} \rightarrow \mathcal{K}$ with respect to a triangulation $\Delta^{\prime}$ obtained from $\Delta$ by finitely many barycentric subdivisions such that $g$ is homotopic to $f$ and the image of $g$ is contained in $\operatorname{st}\left(v_{0}\right)$.

Now we return to the arc complex $\mathcal{A}_{g, n}$ of a compact orientable surface $\Sigma_{g, n}$ of genus $g$ with $n$ holes, where $n \geq 3$ if $g=0$ and $n \geq 1$ if $g \geq 1$. It is a standard fact that any collection of isotopy classes of essential arcs in $\Sigma_{g, n}$ can be realized by a collection of representative arcs having pairwise minimal intersection. In particular, for a collection $\left\{v_{0}, v_{1}, \ldots, v_{k}\right\}$ of vertices of $\mathcal{A}_{g, n}$, if $v_{i}$ and $v_{j}$ are joined by an edge for each $0 \leq i<j \leq k$, then $\left\{v_{0}, v_{1}, \ldots, v_{k}\right\}$ spans a $k$-simplex. Thus, we have the following:

Lemma 1.2. The arc complex $\mathcal{A}_{g, n}$ is a flag complex, and any full subcomplex of $\mathcal{A}_{g, n}$ is also a flag complex.

Let $\alpha$ and $\alpha_{0}$ be essential arcs on the surface $\Sigma_{g, n}$ that intersect each other transversely and minimally. A component $\beta$ of $\alpha_{0}$ cut off by $\alpha \cap \alpha_{0}$ is said to be outermost if $\beta \cap \alpha$ consists of a single point. We note that there exist exactly two such subarcs of $\alpha_{0}$. The intersection $\beta \cap \alpha$ cuts $\alpha$ into two subarcs $\beta^{\prime}$ and $\beta^{\prime \prime}$. We call the two new arcs $\alpha^{\prime}=\beta \cup \beta^{\prime}$ and $\alpha^{\prime \prime}=\beta \cup \beta^{\prime \prime}$ the arcs obtained from $\alpha$ by surgery along $\beta$. We observe that by a small isotopy $\alpha^{\prime}$ and $\alpha^{\prime \prime}$ are disjoint from $\alpha$, and $\left|\alpha_{0} \cap \alpha^{\prime}\right|<\left|\alpha_{0} \cap \alpha\right|$ and $\left|\alpha_{0} \cap \alpha^{\prime \prime}\right|<\left|\alpha_{0} \cap \alpha\right|$ since the intersection $\beta \cap \alpha$ is no longer counted.

Theorem 1.3. Any full subcomplex $\mathcal{A}$ of $\mathcal{A}_{g, n}$ satisfying the following property is contractible.

Surgery Property: Let $\alpha$ and $\alpha_{0}$ be representative arcs of vertices of $\mathcal{A}$ that intersect each other transversely an minimally. If $\alpha \cap \alpha_{0} \neq \emptyset$, then at least one of the two arcs obtained from $\alpha$ by surgery along an outermost subarc of $\alpha_{0}$ cut off by $\alpha \cap \alpha_{0}$ represents a vertex of $\mathcal{A}$.

Proof. Fix a base vertex $v_{0}$ of $\mathcal{A}$. By Lemmas 1.1 and 1.2 it suffices to find a remoteness function for $v_{0}$ that admits a blocking function. For each vertex $v$ of $\mathcal{A}$, define $r(v)$ to be the minimal cardinality of the intersection $\alpha \cap \alpha_{0}$, where $\alpha$ and $\alpha_{0}$ are representative arcs of $v$ and $v_{0}$, respectively. By definition, $r$ is a remoteness function for $v_{0}$. 

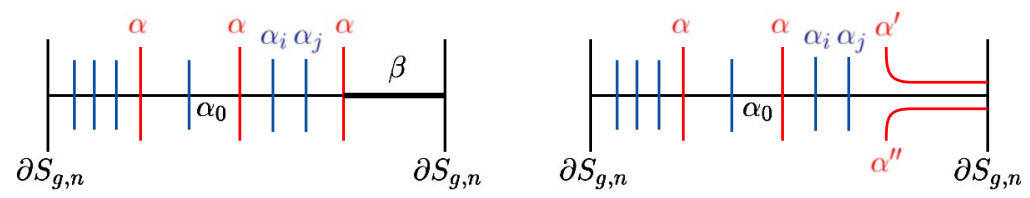

Figure 2
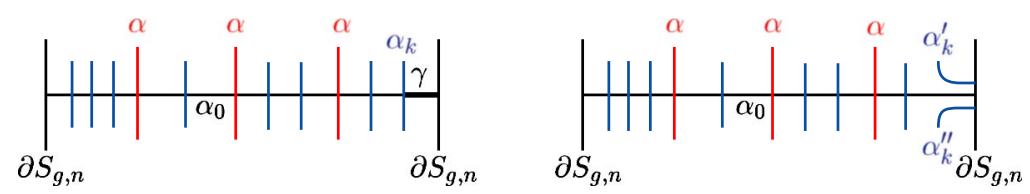

Figure 3

Let $(X, v)$ be an adjacent pair in $\mathcal{A}$, where $r(v)>0$ and $X=\left\{v_{1}, v_{2}, \ldots, v_{n}\right\}$. Choose representative $\operatorname{arcs} \alpha, \alpha_{1}, \alpha_{2}, \ldots, \alpha_{n}$, and $\alpha_{0}$ of $v, v_{1}, v_{2}, \ldots, v_{n}$, and $v_{0}$, respectively, so that they have transverse and pairwise minimal intersection, and every crossing is a double point. Since $r(v)>0$, we have $\alpha \cap \alpha_{0} \neq \emptyset$. Among the two subarcs of $\alpha_{0}$ cut off by $\alpha \cap \alpha_{0}$, choose one, say $\beta$, so that the cardinality of $\beta \cap\left(\alpha_{1} \cup \alpha_{2} \cup \cdots \cup \alpha_{n}\right)$ is minimal, and then denote this cardinality by $b_{0}=b_{0}(\alpha$, $\left.\alpha_{1}, \alpha_{2}, \ldots, \alpha_{n}, \alpha_{0}\right)$. We define $b(X, v)$ to be the minimal number of $b_{0}$ among all such representative arcs of $v, v_{1}, v_{2}, \ldots, v_{n}$, and $v_{0}$. In the following, we will show that $b$ is a blocking function for $r$.

First, suppose that $b(X, v)=0$. Then by an isotopy we may assume that $\beta \cap\left(\alpha_{1} \cup \alpha_{2} \cup \cdots \cup \alpha_{n}\right)=\emptyset$. By the surgery property at least one of the two arcs obtained from $\alpha$ by surgery along $\beta$, say $\alpha^{\prime}$, represents a vertex $w$ of $\mathcal{A}$. See Figure 2. By the construction, $v$ is adjacent to $w$, and $(X, w)$ is an adjacent pair. Further, we have $r(w) \leq\left|\alpha_{0} \cap \alpha^{\prime}\right|<\left|\alpha_{0} \cap \alpha\right|=r(v)$. Next, suppose that $b(X, v)>0$. We may assume that $\beta \cap\left(\alpha_{1} \cup \alpha_{2} \cup \cdots \cup \alpha_{n}\right)=b(X, v)$ by an isotopy. Let $\gamma$ be the outermost subarc of $\alpha_{0}$ cut off by $\alpha_{1} \cup \alpha_{2} \cup \cdots \cup \alpha_{n}$ that is contained in $\beta$. The point $\left(\alpha_{1} \cup \alpha_{2} \cup \cdots \cup \alpha_{n}\right) \cap \gamma$ is contained in $\alpha_{k}$ for some $k \in\{1,2, \ldots, n\}$. Then again by the surgery property at least one of the arcs obtained from $\alpha_{k}$ by surgery along $\gamma$ represents a vertex, say $w^{\prime}$, of $\mathcal{A}$. See Figure 3 . By the construction we have $r\left(w^{\prime}\right)<r\left(v_{k}\right), b\left(X \backslash\left\{v_{k}\right\} \cup\left\{w^{\prime}\right\}, v\right)<b(X, v)$, and each element $x$ of $X$ adjacent to $v_{k}$ is also adjacent to $w^{\prime}$. This completes the proof.

Let $n \geq 2$. We denote by $\mathcal{A}_{g, n}^{*}$ the full subcomplex of $\mathcal{A}_{g, n}$ spanned by the vertices represented by simple arcs connecting the different components of the boundary of $\Sigma_{g, n}$. It is easy to verify that the arc complex $\mathcal{A}_{g, n}$ itself and the subcomplex $\mathcal{A}_{g, n}^{*}$ satisfy the surgery property. Thus we have the following.

COROLlary 1.4. The complexes $\mathcal{A}_{g, n}$ and $\mathcal{A}_{g, n}^{*}$ are contractible.

We end the section with the following lemma for later use. 
Lemma 1.5. The dimension of the complex $\mathcal{A}_{g, 2}^{*}$ is $4 g-1$.

Proof. Let $A=\left\{\alpha_{1}, \alpha_{2}, \ldots, \alpha_{n}\right\}$ be a maximal set of mutually disjoint, mutually nonisotopic simple arcs connecting the different components of the boundary of $\Sigma_{g, 2}$. By contracting each of these boundary components of $\Sigma_{g, 2}$ into a point we get a closed orientable surface $\Sigma$ of genus $g$ with two dots, say $v^{+}$and $v^{-}$. On this surface, each of the arcs of $A$ connects the two dots. Hence, $A$ decomposes $\Sigma$ into cubes with the vertex sets $\left\{v^{+}, v^{-}\right\}$. Now, the assertion follows easily from Euler characteristic considerations.

\section{Sphere Complexes}

Let $(V, W ; \Sigma)$ be a Heegaard splitting of genus $g \geq 2$ of a closed orientable 3manifold $M$. A separating sphere $P$ embedded in $M$ is called a Haken sphere for the spitting if it intersects $\Sigma$ transversely in a single essential simple closed curve. Since $P$ is separating in $M$, the curve $P \cap \Sigma$ is separating in $\Sigma$. Two Haken spheres $P$ and $Q$ are said to be equivalent if $P \cap \Sigma$ and $Q \cap \Sigma$ are isotopic in $\Sigma$. We denote by $\mu=\mu(V, W ; \Sigma)$ the minimal cardinality of $P \cap Q \cap \Sigma$, where $P$ and $Q$ vary over all pairwise nonequivalent Haken spheres for $(V, W ; \Sigma)$. We note that $\mu$ is a nonnegative even number. It was shown in [23] that $\mu(V, W ; \Sigma)=$ 4 when the genus of the splitting is 2 . When the given splitting $(V, W ; \Sigma)$ admits Haken spheres, the sphere complex for the splitting is defined as in Introduction, which we will denote by $\mathcal{H}=\mathcal{H}(V, W ; \Sigma)$.

Given a closed orientable surface $\Sigma$ of genus $g \geq 1$, the curve complex $\mathcal{C}_{g}$ is defined to be the simplicial complex whose vertices are isotopy classes of simple closed curves in $\Sigma$ and whose $k$-simplices are collections of $k+1$ vertices represented by pairwise disjoint and nonisotopic curves in $\Sigma$. It is known that the curve complex $\mathcal{C}_{g}$ is connected and $(3 g-4)$-dimensional. When the surface is the Heegaard surface of a Heegaard splitting $(V, W ; \Sigma)$ of a 3-manifold, we have the two full subcomplexes $\mathcal{D}_{V}$ and $\mathcal{D}_{W}$ of $\mathcal{C}_{g}$, which are spanned by the vertices of the simple closed curves bounding disks in $V$ and $W$, respectively. Then we define the Hempel distance of the splitting to be the minimal simplicial distance in $\mathcal{C}_{g}$ between the two subcomplexes $\mathcal{D}_{V}$ and $\mathcal{D}_{W}$. That is, the minimal number of edges among all the paths in $\mathcal{C}_{g}$ from a vertex of $\mathcal{D}_{V}$ to a vertex of $\mathcal{D}_{W}$. We refer the reader to [14] for details on the Hempel distance. In the case of genus-1 Heegaard splitting for a 3-manifold, we have that the Hempel distance is 0 if the manifold is $S^{2} \times S^{1}$ and $\infty$ otherwise.

Let $(V, W ; \Sigma)$ be a Heegaard splitting of a closed orientable 3-manifold $M$. A nonseparating disk $E_{0}$ in $V$ is called a reducing disk if $\partial E_{0}$ bounds a disk in $W$. We note that if there exists a reducing disk in $M$, then $M$ has an $S^{2} \times S^{1}$ summand for its prime decomposition, and vice versa by Waldhausen's uniqueness of Heegaard splittings of $S^{2} \times S^{1}$ [25] and Haken's lemma [12]. Given any simple closed curve $\gamma$ in $\Sigma$ intersecting $\partial E_{0}$ transversely in a single point, the boundary of $\operatorname{Nbd}\left(\partial E_{0} \cup \gamma ; \Sigma\right)$ is a separating simple closed curve in $\Sigma$ that bounds a disk in each of $V$ and $W$. Thus, if the genus of the splitting is greater than 1 , such a 


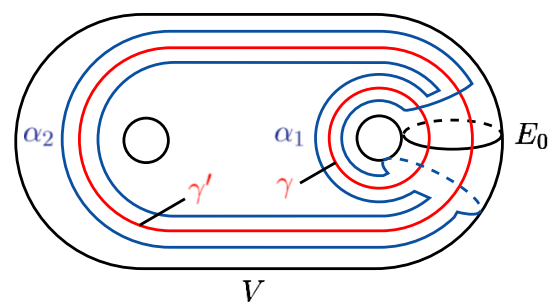

Figure 4

simple closed curve $\gamma$ determines a Haken sphere $P=P(\gamma)$ for the splitting, the union of those two disks in $V$ and $W$.

LemMa 2.1. Let $(V, W ; \Sigma)$ be a genus-g Heegaard splitting of a 3-manifold $M$, where $g \geq 2$. Let $E_{0}$ be a reducing disk in $V$. Let $\gamma$ and $\gamma^{\prime}$ be simple closed curves each of which intersects $\partial E_{0}$ transversely in a single point. Let $P=P(\gamma)$ and $P^{\prime}=P\left(\gamma^{\prime}\right)$ be Haken spheres determined by the curves $\gamma$ and $\gamma^{\prime}$, respectively. Then there exists an orientation-preserving homeomorphism of the manifold $M$ onto itself that maps $P$ to $P^{\prime}$ while preserving each of $V$ and $W$ setwise.

Proof. If $\gamma^{\prime}$ is isotopic to $\gamma$ up to Dehn twists about $\partial E_{0}$, then $P$ and $P^{\prime}$ are equivalent, and thus there is nothing to prove. Otherwise, suppose first that $\gamma^{\prime}$ is disjoint from $\gamma$ up to Dehn twists about $\partial E_{0}$. We may assume without loss of generality that $\gamma^{\prime}$ itself is disjoint from $\gamma$ because Dehn twists about $\partial E_{0}$ do not change the equivalence class of $P^{\prime}$. The boundary of $\operatorname{Nbd}\left(\partial E_{0} ; \Sigma\right)$ consists of two simple closed curves $\delta_{1}$ and $\delta_{2}$. For each $i \in\{1,2\}$, the intersection of $\delta_{i}$ and $\gamma \cup \gamma^{\prime}$ cuts $\delta_{i}$ into two $\operatorname{arcs} \delta_{i, 1}$ and $\delta_{i, 2}$. We set $\alpha_{i}=\left(\left(\gamma \cup \gamma^{\prime}\right) \backslash \operatorname{Nbd}\left(\partial E_{0} ; \Sigma\right)\right) \cup \delta_{1,1} \cup$ $\delta_{2, i}$. See Figure 4 . We note that the union $\alpha_{1} \cup \alpha_{2}$ bounds proper annuli $A$ and $B$ in $V$ and $W$, respectively. Then a single Dehn twist about the annulus $A$, which extends to the Dehn twist about the torus $A \cup B$, is the required homeomorphism of $M$. Here we remark that this homeomorphism is actually a "sliding" of a foot of the 1-handle of each of $V$ and $W$ whose belt sphere is $\partial E_{0}$.

The general case follows from the connectivity of the arc complex as follows. The simple closed curve $\partial E_{0}$ cuts $\Sigma$ into a genus- $(g-1)$ surface $\Sigma_{g-1,2}$ with two holes $\partial E_{0}^{+}$and $\partial E_{0}^{-}$coming from $\partial E_{0}$. On the surface $\Sigma_{g-1,2}, \gamma$ and $\gamma^{\prime}$ are simple arcs $\beta$ and $\beta^{\prime}$ connecting the two holes. Since the complex $\mathcal{A}_{g-1,2}^{*}$ is connected by Corollary 1.4 , there exists a sequence $\beta=\beta_{1}, \beta_{2}, \ldots, \beta_{n}=\beta^{\prime}$ of mutually nonisotopic, essential arcs in $\Sigma_{g-1,2}$ connecting $\partial E_{0}^{+}$and $\partial E_{0}^{-}$ such that $\beta_{i}$ is disjoint from $\beta_{i+1}$ for each $i \in\{1,2, \ldots, n-1\}$. Gluing $\partial E_{0}^{+}$ and $\partial E_{0}^{-}$back, this sequence gives rise to a sequence of simple closed curves $\gamma=\gamma_{1}, \gamma_{2}, \ldots, \gamma_{n}=\gamma^{\prime}$ such that $\gamma_{i+1}$ is disjoint from $\gamma_{i}$ up to Dehn twists about $\partial E_{0}$ for each $i \in\{1,2, \ldots, n-1\}$. Then there exists an orientation-preserving homeomorphism $g_{i}$ of the manifold $M$ onto itself that maps $P\left(\gamma_{i}\right)$ to $P\left(\gamma_{i+1}\right)$ 
while preserving each of $V$ and $W$ setwise. Then the composition $g_{n-1} g_{i-2} \cdots g_{1}$ is the desired homeomorphism.

Let $(V, W ; \Sigma)$ be a genus- $g$ Heegaard splitting of a 3-manifold $M$ with $g \geq 2$. Suppose that there exists a reducing disk $E_{0}$ in $V$. We denote by $\mathcal{H}_{E_{0}}$ the simplicial complex whose vertices are equivalence classes of Haken spheres $P=P(\gamma)$ determined by simple closed curves $\gamma$ in $\Sigma$ intersecting $\partial E_{0}$ transversely in a single point and whose $k$-simplices are collections of $k+1$ vertices represented by pairwise nonequivalent Haken spheres $P\left(\gamma_{0}\right), P\left(\gamma_{1}\right), \ldots, P\left(\gamma_{k}\right)$ such that the minimal cardinality of each $P\left(\gamma_{i}\right) \cap P\left(\gamma_{j}\right) \cap \Sigma$ is 4 for $0 \leq i<j \leq k$ (equivalently the arcs $\gamma_{i}$ and $\gamma_{j}$ are disjoint from each other). We observe that a Haken sphere $P$ represents a vertex of $\mathcal{H}_{E_{0}}$ if and only if $P$ cuts off from $V$ a solid torus whose meridian disk is $E_{0}$. By construction, if $\mu(V, W ; \Sigma)=4$, then the complex $\mathcal{H}_{E_{0}}$ is a full subcomplex of the sphere complex $\mathcal{H}$ of the splitting $(V, W ; \Sigma)$. The following lemma is immediate from the definition of the complex $\mathcal{H}_{E_{0}}$ with Corollary 1.4 and Lemma 1.5.

Lemma 2.2. Let $(V, W ; \Sigma)$ be a genus-g Heegaard splitting of a closed orientable 3-manifold $M$ with $g \geq 2$. Let $E_{0}$ be a reducing disk in $V$. Then the complex $\mathcal{H}_{E_{0}}$ is isomorphic to the complex $\mathcal{A}_{g-1,2}^{*}$, and hence it is a contractible, $(4 g-5)$ dimensional complex.

Proposition 2.3. Let $(V, W ; \Sigma)$ be a genus-g Heegaard splitting of a closed orientable 3-manifold $M$ with $g \geq 2$. Suppose that there exists a unique reducing disk $E_{0}$ in $V$ and also that $\mu(V, W ; \Sigma)>0$. Then the sphere complex $\mathcal{H}$ for the splitting $(V, W ; \Sigma)$ coincides with the complex $\mathcal{H}_{E_{0}}$.

Proof. Let $P$ be a Haken sphere for the splitting $(V, W ; \Sigma)$ intersecting $E_{0}$ transversely and minimally. Suppose that $P \cap E_{0} \neq \emptyset$. At least one, say $M_{1}$, of the closed 3-manifolds $M_{1}$ and $M_{2}$ obtained by cutting $M$ along $P$ and then capping off the resulting boundary spheres by adding 3-balls has an $S^{2} \times S^{1}$ summand for its prime decomposition. Then as mentioned in the last paragraph before Lemma 2.1, the $V$ part of the Heegaard splitting of $M_{1}$ naturally induced from $(V, W ; \Sigma)$ contains a reducing disk, which gives rise to a reducing disk of $V$ that is not isotopic to $E_{0}$. This contradicts the uniqueness of $E_{0}$. Therefore, any Haken sphere is disjoint from the reducing disk $E_{0}$. It suffices to show that $P$ cuts off a solid torus from $V$ whose meridian disks is $E_{0}$. Suppose not, that is, the component $\Sigma^{\prime}$ of $\Sigma$ cut off by $P \cap \Sigma$ containing $\partial E_{0}$ is a compact surface of genus at least 2 . Then we can choose a simple closed curve $\gamma$ in $\Sigma^{\prime}$ intersecting $\partial E_{0}$ transversely in a single point such that the Haken sphere $Q=Q(\gamma)$ is disjoint from and is not equivalent to $P$. We have then $0<\mu(V, W ; \Sigma) \leq|P \cap Q \cap \Sigma|=0$, a contradiction.

Now we will construct (infinitely many) Heegaard splittings $(V, W ; \Sigma)$ satisfying the conditions in Proposition 2.3, that is, 
- there exists a unique reducing disk in $V$; and

- $\mu(V, W ; \Sigma)>0$.

Let $\left(V_{1}, W_{1} ; \Sigma_{1}\right)$ and $\left(V_{2}, W_{2} ; \Sigma_{2}\right)$ be genus- $g_{1}$ and genus- $g_{2}$ Heegaard splittings for 3-manifolds $M_{1}$ and $M_{2}$, respectively. Let $B_{1}$ and $B_{2}$ be 3-balls in $M_{1}$ and $M_{2}$ that intersect the Heegaard surfaces $\Sigma_{1}$ and $\Sigma_{2}$ in a single disk, respectively. Removing the interiors of $B_{1}$ and $B_{2}$, and identifying $\partial B_{1}$ and $\partial B_{2}$, we can construct a genus- $\left(g_{1}+g_{2}\right)$ Heegaard splitting $(V, W ; \Sigma)$ for the connected sum $M=M_{1} \# M_{2}$ such that $V$ and $W$ are considered as boundary connected sums of $V_{1}$ and $V_{2}$ and of $W_{1}$ and $W_{2}$, respectively. We call the splitting $(V, W ; \Sigma)$ a Heegaard splitting for $M$ obtained from $\left(V_{1}, W_{1} ; \Sigma_{1}\right)$ and $\left(V_{2}, W_{2} ; \Sigma_{2}\right)$. We note that the sphere $P=\partial B_{1}=\partial B_{2}$ is a Haken sphere for the splitting $(V, W ; \Sigma)$. In the remaining of the section, we always assume the following:

- $\left(V_{1}, W_{1} ; \Sigma_{1}\right)$ is a genus- $(g-1)$ Heegaard splitting for a closed orientable 3manifold $M_{1}$ with $g \geq 2$, and $\left(V_{2}, W_{2} ; \Sigma_{2}\right)$ is the genus-1 Heegaard splitting for $S^{2} \times S^{1}$.

- $(V, W ; \Sigma)$ is a genus- $g$ Heegaard splitting for $M=M_{1} \#\left(S^{2} \times S^{1}\right)$ obtained from $\left(V_{1}, W_{1} ; \Sigma_{1}\right)$ and $\left(V_{2}, W_{2} ; \Sigma_{2}\right)$ by the above construction, and $P=$ $\partial B_{1}=\partial B_{2}$ is the Haken sphere for the splitting $(V, W ; \Sigma)$.

- $E_{0}$ and $E_{0}^{\prime}$ with $\partial E_{0}=\partial E_{0}^{\prime}$ are meridian disks of the solid tori $V_{2}$ and $W_{2}$, respectively, which are reducing disks for the splitting $(V, W ; \Sigma)$.

We start with the following two lemmas.

LEMMA 2.4. Let $\delta$ be an essential simple closed curve in $\Sigma$ that is disjoint from and not isotopic to $\partial E_{0}$. Suppose that $\delta$ does not cut off from $\Sigma$ a torus with one hole containing $\partial E_{0}$. If $\delta$ bounds disks in $V$ and $W$ simultaneously, then the Hempel distance of the splitting $\left(V_{1}, W_{1} ; \Sigma\right)$ is 0 .

From the lemma it is easy to see that if the Hempel distance of the splitting $\left(V_{1}, W_{1} ; \Sigma_{1}\right)$ is at least 1 and if $E$ is an essential nonseparating disk in $V$ that is disjoint from and not isotopic to $E_{0}$, then $E$ cannot be a reducing disk for the splitting $(V, W ; \Sigma)$.

Proof of Lemma 2.4. Suppose that $\delta$ in $\Sigma$ bounds disks both in $V$ and $W$. We want to find an essential simple closed curve in $\Sigma_{1}$ that bounds disks both in $V_{1}$ and $W_{1}$.

Among the simple closed curves in $\Sigma$ that intersect $\partial E_{0}$ transversely in a single point, choose one, say $\gamma$, so that $\gamma$ intersects $\delta$ minimally. Then either $\gamma$ is disjoint from $\delta$ or $\gamma$ intersects $\delta$ in a single point. (If $\delta$ is nonseparating and $\delta \cup \partial E_{0}$ is separating in $\Sigma$, then we have to choose such a curve $\gamma$ so that $\gamma$ intersects $\delta$ in a single point. Otherwise, we can choose $\gamma$ disjoint from $\delta$.) Let $P(\gamma)$ be the Haken sphere determined by $\gamma$, that is, $P(\gamma) \cap \Sigma$ is the boundary of $\operatorname{Nbd}\left(\partial E_{0} \cup \gamma ; \Sigma\right)$. Applying Lemma 2.1, we may assume that the Haken sphere $P\left(=\partial B_{1}=\partial B_{2}\right)$ equals $P(\gamma)$ and that $\operatorname{Nbd}\left(E_{0} \cup \gamma ; V\right)$ and $\operatorname{Nbd}\left(E_{0}^{\prime} \cup \gamma ; W\right)$ are solid tori $V_{2}$ and $W_{2}$, respectively, with the interior of the 3-ball $B_{2}$ removed. 


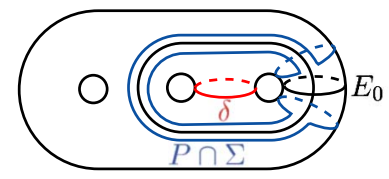

(i)

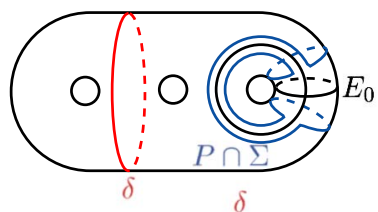

(ii)

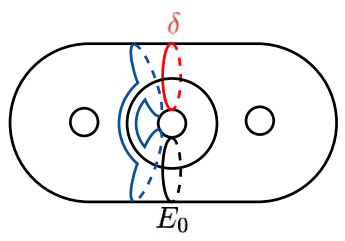

(iii)

Figure 5

If $\gamma$ is disjoint from $\delta$, then by isotopy we may assume that $\delta$ lies in $\Sigma_{1}$ outside the disk $B_{1} \cap \Sigma_{1}$ and that the union of the disk in $V$ and that in $W$ bounded by $\delta$ are disjoint from and not isotopic to $P$. Apparently, $\delta$ remains to be essential in $\Sigma_{1}$. See Figure 5(i) and (ii). Thus, the Hempel distance of $\left(V_{1}, W_{1} ; \Sigma_{1}\right)$ is 0 in this case. If $\gamma$ intersects $\delta$ in a single point, then we cannot say that $\delta$ lies in $\Sigma_{1}$. But by isotopy we may assume that the boundary of $\operatorname{Nbd}\left(\partial E_{0} \cup \delta \cup \gamma ; \Sigma\right)$, which consists of two simple closed curves, lies in $\Sigma_{1}$ outside the disk $B_{1} \cap \Sigma_{1}$. Any of the two simple closed curves bound disks in $V$ and $W$, which can be isotoped to be disjoint from $P$. Since $\delta$ is not isotopic to $\partial E_{0}$ in $\Sigma$, each of these simple closed curves is essential in $\Sigma_{1}$. See Figure 5(iii). Again, the Hempel distance of $\left(V_{1}, W_{1} ; \Sigma\right)$ is 0 .

LEMmA 2.5. Let $\delta_{1}$ and $\delta_{2}$ be disjoint, nonseparating simple closed curves in $\Sigma$ each of which is disjoint from and not isotopic to $\partial E_{0}$. If $\delta_{1}$ bounds a disk in $V$ and $\delta_{2}$ bounds a disk in $W$, then the Hempel distance of the splitting $\left(V_{1}, W_{1} ; \Sigma\right)$ is at most 1 .

Proof. The argument will be very similar to the proof of Lemma 2.4. We note that $\delta_{1}$ is possibly isotopic to $\delta_{2}$. Suppose that $\delta_{1}$ and $\delta_{2}$ bound disks in $V$ and $W$, respectively. We want to find two disjoint, essential simple closed curves in $\Sigma_{1}$ such that one bounds a disk in $V_{1}$ and the other in $W_{1}$. Among the simple closed curves in $\Sigma$ that intersect $\partial E_{0}$ transversely in a single point, choose one, say $\gamma$, so that $\gamma$ intersects $\delta_{1} \cup \delta_{2}$ minimally. We may assume that the Haken sphere $P$ equals $P(\gamma)$ as in the proof of Lemma 2.4. For each $i \in\{1,2\}, \delta_{i}$ is disjoint from $\gamma$ or intersects $\gamma$ in a single point, and hence we have four cases.

If each of $\delta_{1}$ and $\delta_{2}$ is disjoint from $\gamma$, then by isotopy we may assume that $\delta_{1}$ and $\delta_{2}$ lie inside $\Sigma_{1}$ as disjoint, essential simple closed curves, and these bound disks inside $V_{1}$ and $W_{1}$, respectively. See Figure 6(i).

If one of them, say $\delta_{1}$, intersects $\gamma$ in a single point and the other one $\delta_{2}$ is disjoint from $\gamma$, then consider the boundary of $\operatorname{Nbd}\left(\partial E_{0} \cup \delta_{1} \cup \gamma ; \Sigma\right)$, which consists of two simple closed curves. By isotopy we may assume that both of the two simple closed curves lie inside $\Sigma_{1}$ as essential simple closed curves and bound disks in $V_{1}$, whereas $\delta_{2}$ is an essential simple closed curve in $\Sigma_{1}$ disjoint from these curves and bounding a disk in $W_{1}$. See Figure 6(ii). 


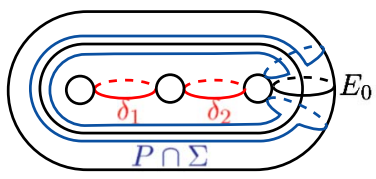

(i)

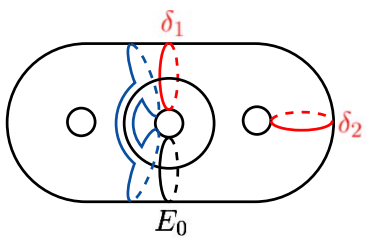

(ii)

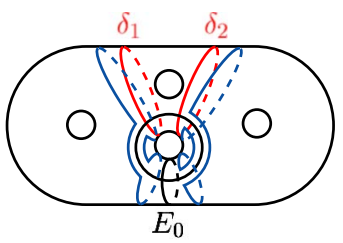

(iii)

Figure 6

Finally, if each of $\delta_{1}$ and $\delta_{2}$ intersects $\gamma$ in a single point, then consider the boundary of $\operatorname{Nbd}\left(\partial E_{0} \cup \delta_{1} \cup \delta_{2} \cup \gamma ; \Sigma\right)$, which consists of three simple closed curves. By isotopy again, we may assume that all the three curves lie in $\Sigma_{1}$. Among the three curves, one is a component of the boundary of $\operatorname{Nbd}\left(\partial E_{0} \cup \delta_{1} \cup \gamma ; \Sigma\right)$, which bounds a disk in $V_{1}$, and another one is a component of the boundary of $\operatorname{Nbd}\left(\partial E_{0} \cup \delta_{2} \cup \gamma ; \Sigma\right)$, which bounds a disk in $W_{1}$. (The third one may bound a disk neither in $V_{1}$ nor in $W_{1}$.) See Figure 6(iii). Again, these two simple closed curves are essential in $\Sigma_{1}$. Therefore, in any of four cases, the Hempel distance of the splitting $\left(V_{1}, W_{1} ; \Sigma\right)$ is at most 1 .

Let $D$ and $E$ be essential disks in the handlebody $V$ that intersect each other transversely and minimally. A subdisk $\Delta$ of $D$ cut off by $D \cap E$ is said to be outermost if $\Delta \cap E$ is a single arc. For an outermost subdisk $\Delta$ of $D$ cut off by $D \cap E$, the arc $\Delta \cap E$ cuts $E$ into two disks, say $E^{\prime}$ and $E^{\prime \prime}$. We call the two disks $E_{1}=E^{\prime} \cup \Delta$ and $E_{2}=E^{\prime \prime} \cup \Delta$ the disks obtained from $E$ by surgery along $\Delta$. Both of $E_{1}$ and $E_{2}$ can be isotoped to be disjoint from $E$. By an elementary argument of the reduced homology group $H_{2}(V, \partial V ; \mathbb{Z})$ we can check easily that at least one of $E_{1}$ and $E_{2}$ is nonseparating if $E$ is nonseparating.

For any simple closed curves $\gamma$ and $\delta$ in the surface $\Sigma$ that intersect each other transversely and minimally in at least two points, we can define similarly the two simple closed curves $\gamma_{1}$ and $\gamma_{2}$ obtained from $\gamma$ by surgery along an innermost subarc of $\delta$ cut off by $\gamma \cap \delta$. Here an innermost subarc, say $\delta^{\prime}$, is a component of $\delta$ cut off by $\gamma \cap \delta$ that meets $\gamma$ only in its endpoints and cuts $\gamma$ into two arcs, say $\gamma^{\prime}$ and $\gamma^{\prime \prime}$. Then $\gamma_{1}=\gamma^{\prime} \cup \delta^{\prime}$ and $\gamma_{2}=\gamma^{\prime \prime} \cup \delta^{\prime}$. If the subarc $\delta^{\prime}$ meets $\gamma$ from the same side, then both of $\gamma_{1}$ and $\gamma_{2}$ can be isotoped to be disjoint from $\gamma$. We also see that if $\gamma$ is nonseparating, then at least one of $\gamma_{1}$ and $\gamma_{2}$ is nonseparating by an elementary argument of $H_{2}(\Sigma ; \mathbb{Z})$.

Proposition 2.6. Suppose that the Hempel distance of the splitting $\left(V_{1}, W_{1} ; \Sigma\right)$ is at least 2. Then we have the following:

(1) $E_{0}$ is the unique reducing disk in $V$, and

(2) $\mu(V, W ; \Sigma)>0$.

Proof. Statement (2) is easy to verify. In fact, if $\mu(V, W ; \Sigma)=0$, then we might find a Haken sphere for the splitting $\left(V_{1}, W_{1} ; \Sigma_{1}\right)$, and hence the Hempel distance of $\left(V_{1}, W_{1} ; \Sigma_{1}\right)$ would be 0 , a contradiction. 

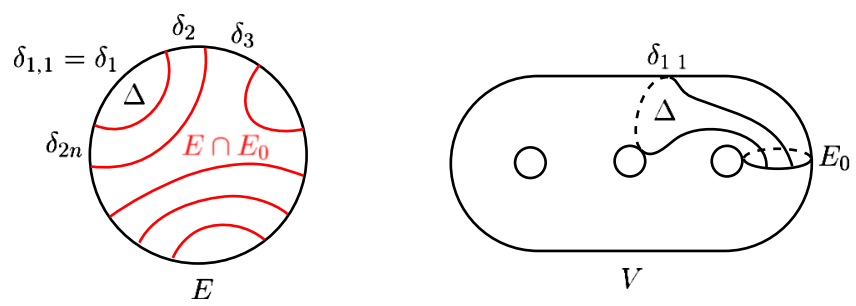

Figure 7

We prove (1). It is proved in [6] that (1) is true when $M_{1} \cong S^{3}$ and $g=2$. In the following, we will assume that $M_{1} \nsucceq S^{3}$ and $g>2$. Let $E$ be an essential nonseparating disk in $V$ that is not isotopic to $E_{0}$. We may assume that $E$ intersects $E_{0}$ transversely and minimally. If $E$ is disjoint from $E_{0}$, then $E$ is not a reducing disk by Lemma 2.4. Suppose that $E$ intersects $E_{0}$. Then $\partial E_{0}$ cuts $\partial E$ into $2 n$ $(n \geq 1)$ simple arcs $\delta_{1}, \delta_{2}, \ldots, \delta_{2 n}$. We divide the collection of these arcs into two subcollections as

$$
\left\{\delta_{1}, \delta_{2}, \ldots, \delta_{2 n}\right\}=\left\{\delta_{1,1}, \delta_{1,2}, \ldots, \delta_{1, n_{1}}\right\} \sqcup\left\{\delta_{2,1}, \delta_{2,2}, \ldots, \delta_{2, n_{2}}\right\},
$$

where each of the arcs $\delta_{1, i}$ meets $\partial E_{0}$ from the same side, whereas each of $\delta_{2, j}$ does from the opposite sides. We may assume without loss of generality that there exists an outermost subdisk $\Delta$ of $E$ cut off by $E \cap E_{0}$ such that $\delta_{1,1} \subset \partial \Delta$. See Figure 7.

Let $\left\{E_{0}^{\prime}, D_{1}^{\prime}, D_{2}^{\prime}, \ldots, D_{g-1}^{\prime}\right\}$ be a complete system of meridian disks of $W$, where $\partial E_{0}^{\prime}=\partial E_{0}$. Fix orientations of the boundary circles $\partial E_{0}^{\prime}$ and $\partial D_{1}^{\prime}, \partial D_{2}^{\prime}, \ldots, \partial D_{g-1}^{\prime}$ and assign symbols $x$ and $y_{1}, y_{2}, \ldots, y_{g-1}$ on the circles, respectively. Then any oriented simple closed curve $\delta$ in $\Sigma$ intersecting the boundary circles transversely determines a word $w(\delta)$ on $\left\{x, y_{1}, y_{2}, \ldots, y_{g-1}\right\}$ that can be read off from the intersections of $\delta$ with the circles. This word determines an element of the free group $\pi_{1}(W)=\left\langle x, y_{1}, y_{2}, \ldots, y_{g-1}\right\rangle$ represented by $\delta$. Let $E_{1}$ and $E_{2}$ be the disks obtained from $E_{0}$ by surgery along $\Delta$. Since $E_{0}$ is nonseparating, at least one of the two, say $E_{1}$, is nonseparating. By a small isotopy we assume that $E_{1}$ is disjoint from $E_{0}$.

CLAIM 1. The word $w\left(\delta_{1,1}\right)$ on $\left\{y_{1}, y_{2}, \ldots, y_{g-1}\right\}$ read off by the interior of the arc $\delta_{1,1}$ represents a nontrivial element of $\pi_{1}(W)=\left\langle x, y_{1}, y_{2}, \ldots, y_{g-1}\right\rangle$.

Proof. The disk $E_{1}$ is nonseparating, disjoint from $E_{0}$, and not isotopic to $E_{0}$, and hence, by Lemma 2.4, it is not a reducing disk. That is, $\partial E_{1}$ does not bound a disk in $W$. Thus, by the loop theorem $w\left(\partial E_{1}\right)=w\left(\delta_{1,1}\right)$ determines a nontrivial element of $\pi_{1}(W)$.

Claim 2. The word $w\left(\delta_{1, i}\right)$ on $\left\{y_{1}, y_{2}, \ldots, y_{g-1}\right\}$ read off by the interior of the arc $\delta_{1, i}\left(2 \leq i \leq n_{1}\right)$ represents a nontrivial element of $\pi_{1}(W)=$ $\left\langle x, y_{1}, y_{2}, \ldots, y_{g-1}\right\rangle$. 

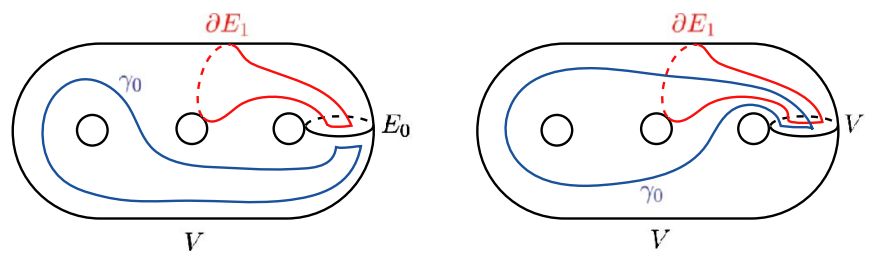

Figure 8

Proof. The arc $\delta_{1, i}$ is an innermost subarc of $\partial E$ cut off by $\partial E \cap \partial E_{0}$. One of the two simple closed curves obtained from $\partial E_{0}$ by surgery along $\delta_{1, i}$ is a nonseparating curve, which we denote by $\gamma_{0}$. By a small isotopy we may assume that $\gamma_{0}$ is disjoint from $\partial E_{0}$. Further, it is easy to see that $\gamma_{0}$ intersects $\partial E_{1}$ transversely at most once. See Figure 8 . If $\gamma_{0}$ does not bound a disk in $W$, then by the loop theorem the word $w\left(\gamma_{0}\right)=w\left(\delta_{1, i}\right)$ determines a nontrivial element of $\pi_{1}(W)$, so we are done. Suppose for a contradiction that $\gamma_{0}$ bounds a disk in $W$. If $\gamma_{0}$ is disjoint from $\partial E_{1}$, then the Hempel distance of the splitting $\left(V_{1}, W_{1} ; \Sigma\right)$ is at most 1 by Lemma 2.5, which is a contradiction. If $\gamma_{0}$ intersects $\partial E_{1}$ in a single point, then the boundary $\delta$ of $\operatorname{Nbd}\left(\gamma_{0} \cup \partial E_{1} ; \Sigma\right)$ is an essential, separating simple closed curve, which is disjoint from $\gamma_{0}$ and bound disks both in $V$ and $W$. The curve $\delta$ cannot cut off from $\Sigma$ a torus with one hole containing $\partial E_{0}$ since otherwise $M_{1} \cong S^{3}$ and $g=2$, a contradiction. Thus, by Lemma 2.4 the Hempel distance of the splitting $\left(V_{1}, W_{1} ; \Sigma\right)$ is 0 by Lemma 2.5 , which is also a contradiction.

Now we can write the word $w(\partial E)$ on $\left\{x, y_{1}, y_{2}, \ldots, y_{g-1}\right\}$ as

$$
x^{\epsilon_{1}} w\left(\delta_{1}\right) x^{\epsilon_{2}} w\left(\delta_{2}\right) x^{\epsilon_{3}} w\left(\delta_{3}\right) \cdots x^{\epsilon_{2 n}} w\left(\delta_{2 n}\right),
$$

where $\epsilon_{k} \in\{-1,1\}$ for $k \in\{1,2, \ldots, 2 n\}$. If $\delta_{k}=\delta_{1, i}$ for some $i \in\left\{1,2, \ldots, n_{1}\right\}$, then $\left\{\epsilon_{k}, \epsilon_{k+1}\right\}=\{-1,1\}$, but $w\left(\delta_{k}\right)$ is nontrivial. If $\delta_{k}=\delta_{2, j}$ for some $j \in$ $\left\{1,2, \ldots, n_{2}\right\}$, then $w\left(\delta_{k}\right)$ is possibly trivial, but $\epsilon_{k}=\epsilon_{k+1}$. (Here $\epsilon_{2 n+1}=\epsilon_{1}$.) This implies that $w(\partial E)$ determines a nontrivial element of $\pi_{1}(W)$, and so $\partial E$ cannot bound a disk in $W$. Thus, $E$ cannot be a reducing disk.

By Lemma 2.2, and Propositions 2.3 and 2.6 we have the main result of the section.

Corollary 2.7. Let $\left(V_{1}, W_{1} ; \Sigma_{1}\right)$ be a genus- $(g-1)$ Heegaard splitting of Hempel distance at least 2 for a closed orientable 3-manifold $M_{1}$, where $g \geq 2$, and let $\left(V_{2}, W_{2} ; \Sigma_{2}\right)$ be the genus-1 Heegaard splitting for $S^{2} \times S^{1}$. If $(V, W ; \Sigma)$ is the splitting for $M_{1} \#\left(S^{2} \times S^{1}\right)$ obtained from $\left(V_{1}, W_{1} ; \Sigma_{1}\right)$ and $\left(V_{2}, W_{2} ; \Sigma_{2}\right)$, then the sphere complex $\mathcal{H}$ for the splitting $(V, W ; \Sigma)$ is isomorphic to the complex $\mathcal{A}_{g-1,2}^{*}$, and hence it is a $(4 g-5)$-dimensional contractible complex.

Recalling that the Hempel distance of the genus-1 Heegaard splitting of $S^{3}$ or a lens space is $\infty$, we also have the following: 
Corollary 2.8. Let $(V, W ; \Sigma)$ be the genus-2 Heegaard splitting for $M_{1} \#\left(S^{2} \times\right.$ $\left.S^{1}\right)$, where $M_{1}$ is $S^{3}$ or a lens space. Then the sphere complex $\mathcal{H}$ for the splitting $(V, W ; \Sigma)$ is a three-dimensional contractible complex.

\section{Goeritz Groups}

Let $M$ be an orientable manifold. Let $X_{1}, X_{2}, \ldots, X_{n}$, and $Y$ be subspaces of $M$. We denote by

$$
\text { Homeo }_{+}\left(M, X_{1}, X_{2}, \ldots, X_{n} \operatorname{rel} Y\right)
$$

the group of orientation-preserving homeomorphisms of $M$ that preserve each of the subspaces $X_{1}, X_{2}, \ldots, X_{n}$ setwise and $Y$ pointwise. We equip this group with the compact-open topology. Let $\operatorname{Homeo}_{0}\left(M, X_{1}, X_{2}, \ldots, X_{n} \operatorname{rel} Y\right)$ be the connected component of $\operatorname{Homeo}_{+}\left(M, X_{1}, X_{2}, \ldots, X_{n}\right.$ rel $\left.Y\right)$ containing the identity. This component is a normal subgroup, and we denote by $\mathrm{MCG}_{+}\left(M, X_{1}, X_{2}, \ldots, X_{n}\right.$ rel $\left.Y\right)$ the quotient group

$$
\text { Homeo }_{+}\left(M, X_{1}, X_{2}, \ldots, X_{n} \operatorname{rel} Y\right) / \operatorname{Homeo}_{0}\left(M, X_{1}, X_{2}, \ldots, X_{n} \operatorname{rel} Y\right) \text {. }
$$

Let $(V, W ; \Sigma)$ be a Heegaard splitting of a closed orientable 3-manifold $M$. We recall that the Goeritz group of the splitting $(V, W ; \Sigma)$ is the group of isotopy classes of the orientation-preserving homeomorphisms of $M$ that preserve $V$ and $W$ setwise. We denote by $\mathcal{G}(V, W ; \Sigma)$ the Goeritz group, which is identified with the quotient group $\mathrm{MCG}_{+}(M, V)$. We note that there are natural injective homomorphisms $\mathrm{MCG}_{+}(V) \rightarrow \mathrm{MCG}_{+}(\Sigma)$ and $\mathrm{MCG}_{+}(W) \rightarrow \mathrm{MCG}_{+}(\Sigma)$, which can be obtained by restricting homeomorphisms of $V$ and $W$ to $\Sigma$, respectively. Once we regard the groups $\mathrm{MCG}_{+}(V)$ and $\mathrm{MCG}_{+}(W)$ as subgroups of $\mathrm{MCG}_{+}(\Sigma)$ with respect to the inclusions, $\mathcal{G}(V, W ; \Sigma)$ is identified with $\operatorname{MCG}_{+}(V) \cap \mathrm{MCG}_{+}(W)$. We also note that the group $\mathcal{G}(V, W ; \Sigma)$ acts on the sphere complex $\mathcal{H}$ of $(V, W ; \Sigma)$ simplicially if the splitting $(V, W ; \Sigma)$ admits Haken spheres.

Namazi [22] showed that if the Hempel distance of the splitting $(V, W ; \Sigma)$ is sufficiently high, then $\mathcal{G}(V, W ; \Sigma)$ is a finite group. Later, Johnson [16] improved this result as follows.

TheOrem 3.1 (Johnson [16]). If the Hempel distance of the splitting $(V, W ; \Sigma)$ is at least 4 , then the group $\mathcal{G}(V, W ; \Sigma)$ is finite.

For Heegaard splittings of low Hempel distance, the situation is much more complicated as mentioned in Introduction.

In this section, we are interested in the Goeritz groups of the Heegaard splittings described in Section 2. Let $(V, W ; \Sigma)$ be a genus- $g$ Heegaard splitting of a closed orientable 3 -manifold $M$, where $g \geq 2$. Suppose that $\mu(V, W ; \Sigma)>0$ and there exists a unique reducing disk $E_{0}$ in $V$. Fix a Haken sphere $P$ for the splitting $(V, W ; \Sigma)$ that represents a vertex of the complex $\mathcal{H}_{E_{0}}$. That is, $P$ is the Haken sphere determined by a simple closed curve in $\Sigma$ intersecting $\partial E_{0}$ in a single point as in Section 2. Then the disk $P \cap V$ cuts off from $V$ a solid torus whose meridian disk is $E_{0}$. 

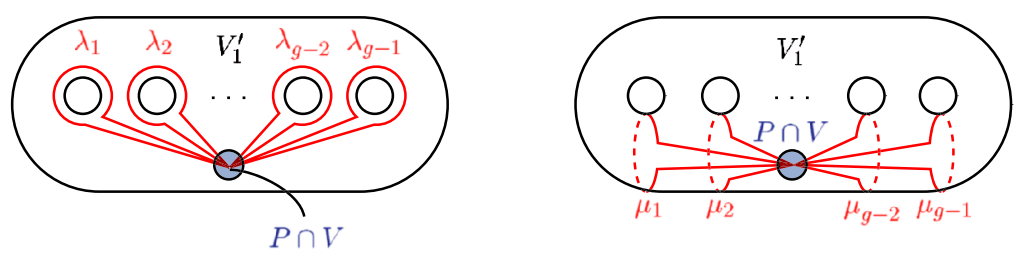

Figure 9

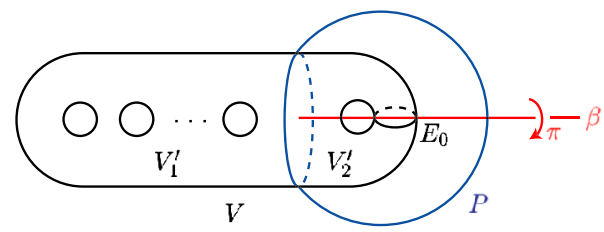

Figure 10

The handlebody $V$ cut off by $P \cap V$ consists of two handlebodies $V_{1}^{\prime}$ and $V_{2}^{\prime}$, and similarly, $W$ cut off by $P \cap W$ consists of $W_{1}^{\prime}$ and $W_{2}^{\prime}$. Gluing 3-balls $B_{1}$ and $B_{2}$ on $V_{1}^{\prime} \cup W_{1}^{\prime}$ and $V_{2}^{\prime} \cup W_{2}^{\prime}$ along $P$, we obtain two Heegaard splittings $\left(V_{1}, W_{1} ; \Sigma_{1}\right)$ and $\left(V_{2}, W_{2} ; \Sigma_{2}\right)$, respectively. We may assume that $\left(V_{2}, W_{2} ; \Sigma_{2}\right)$ is the genus- 1 splitting of $S^{2} \times S^{1}$, whereas $\left(V_{1}, W_{1} ; \Sigma_{1}\right)$ is the genus- $(g-1)$ splitting of a 3-manifold having no $S^{2} \times S^{1}$ summand in its prime decomposition.

Suppose that the Goeritz group $\mathcal{G}\left(V_{1}, W_{1} ; \Sigma_{1}\right)$ is generated by finitely many elements $\omega_{1}, \omega_{2}, \ldots, \omega_{m}$. For each $i \in\{1,2, \ldots, m\}$, the element $\omega_{i}$ has a representative homeomorphism $w_{i} \in \operatorname{Homeo}_{+}\left(M_{1}, V_{1}\right)$ satisfying $\left.w_{i}\right|_{B_{i}}$ is the identity. Thus, there exists an element $\tilde{\omega}_{i}$ of $\mathcal{G}(V, W ; \Sigma)$ represented by a homeomorphism $\tilde{w}_{i} \in \operatorname{Homeo}_{+}(M, V)$ such that $\tilde{w}_{i}(P)=P,\left.\tilde{w}_{i}\right|_{V_{1}^{\prime} \cup W_{1}^{\prime}}=\left.w_{i}\right|_{V_{1}^{\prime} \cup W_{1}^{\prime}}$ and $\left.\tilde{w}_{i}\right|_{V_{2}^{\prime} \cup W_{2}^{\prime}}$ is the identity.

We also define the elements $\lambda_{j}$ and $\mu_{j}$ for each $j \in\{1,2, \ldots, g-1\}$ and the elements $\beta$ and $\epsilon$ of $\mathcal{G}(V, W ; \Sigma)$ as follows. The elements $\lambda_{j}$ and $\mu_{j}$ have representative homeomorphisms $l_{j}$ and $m_{j}$ with $\left.l_{j}\right|_{V_{2}^{\prime} \cup W_{2}^{\prime}}=\left.m_{j}\right|_{V_{2}^{\prime} \cup W_{2}^{\prime}}=\mathrm{id}_{V_{2}^{\prime} \cup W_{2}^{\prime}}$ obtained by pushing $V_{2}^{\prime} \cup W_{2}^{\prime}$ so that $P \cap V$ moves along the arcs depicted in Figure 9 , respectively.

The element $\beta$ is defined by extending a half-Dehn twist about the disk $P \cap V$, and the element $\epsilon$ is defined by extending a Dehn twist about the unique reducing disk $E_{0}$ in $V$. See Figure 10 . Note that all of $\tilde{\omega}_{i}, \lambda_{j}, \mu_{j}, \beta$, and $\epsilon$ preserve the equivalence class of the Haken sphere $P$.

Lemma 3.2. Under this setting, the subgroup of $\mathcal{G}(V, W ; \Sigma)$ consisting of elements that preserve the equivalence class of $P$ is generated by $\tilde{\omega}_{i}, \lambda_{j}, \mu_{j}, \beta$, and $\epsilon$, where $i \in\{1,2, \ldots, m\}$ and $j \in\{1,2, \ldots, g-1\}$.

Proof. Let $l_{j}, m_{j}, b$, and $e$ be representative homeomorphisms of $\lambda_{j}, \mu_{j}, \beta$, and $\epsilon$, respectively, preserving $P$. We may assume that each of $m_{j}, l_{j}$, and $b^{2}$ fixes 
$V_{2}^{\prime} \cup W_{2}^{\prime}$. Let $\varphi$ be any element of $\mathcal{G}(V, W ; \Sigma)$ that preserves the equivalence class of $P$, and let $f \in \mathrm{Homeo}_{+}(M, V)$ be one of its representatives satisfying $f(P)=P$. We will show that $f$ is isotopic to a composition of a finite number of $\tilde{w}_{i}^{ \pm 1}, l_{j}^{ \pm 1}, m_{j}^{ \pm 1}, b^{ \pm 1}$, and $e^{ \pm 1}$ up to an isotopy preserving $V$.

Let $E_{0}^{\prime}$ be an essential disk in $W$ bounded by the unique reducing disk $\partial E_{0}$ in $V$. Composing $f$ with a power of $b$, if necessary, and by an appropriate isotopy preserving $V$, we get a map $f_{1} \in \operatorname{Homeo}_{+}(M, V)$ fixing $E_{0} \cup E_{0}^{\prime}$ and $P$. Moreover, by composing $f_{1}$ with a power of $e$, if necessary, and by an appropriate isotopy preserving $V$, we get a map $f_{2} \in \operatorname{Homeo}_{+}(M, V)$ fixing $E_{0} \cup E_{0}^{\prime}, \partial V_{2}^{\prime}$, and $\partial W_{2}^{\prime}$. Note that the union of $E_{0} \cup E_{0}^{\prime}$ and $\partial V_{2}^{\prime} \cap \partial W_{2}^{\prime}$ cuts $V_{2}^{\prime} \cup W_{2}^{\prime}$ into two 3-balls. Thus, by Alexander's trick, we may assume that $f_{2}$ fixes $V_{2}^{\prime} \cup W_{2}^{\prime}$.

Suppose first that $g \geq 3$. Let $D_{1}$ be the disk $\Sigma_{1} \cap B_{1}$ and choose a point $p_{1}$ in the interior of $D_{1}$. By the Birman exact sequence [2] we have the following commutative diagrams:

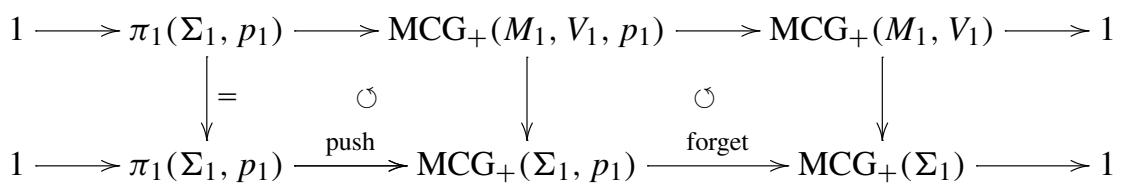

and

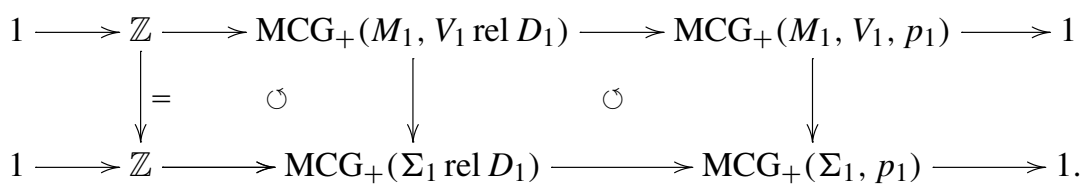

In these diagrams, each vertical arrow is an injective homeomorphism. In the first diagram, the arrow " $\stackrel{\text { push }}{\longrightarrow}$ implies the pushing map, and " $\stackrel{\text { forget }}{\longrightarrow}$ " implies the forgetful map. The group $\mathbb{Z}$ in the second diagram is generated by the Dehn twist about the disk $D_{1}$. See, for instance, [9; 11]. By the assumption the group $\mathrm{MCG}_{+}\left(M_{1}, V_{1}\right)=\mathcal{G}\left(V_{1}, W_{1} ; \Sigma_{1}\right)$ is generated by $\omega_{1}, \omega_{2}, \ldots, \omega_{m}$. The image of $\pi_{1}\left(\partial V_{1}, p_{1}\right)$ in $\mathrm{MCG}_{+}\left(M_{1}, V_{1}, p_{1}\right)$ is the subgroup generated by the elements whose representatives correspond to $\left.l_{j}\right|_{V_{1}^{\prime} \cup W_{1}^{\prime}}$ and $\left.m_{j}\right|_{V_{1}^{\prime} \cup W_{1}^{\prime}}$, where $j \in\{1,2, \ldots, g-1\}$. Moreover, a generator of $\mathbb{Z}$ in the second diagram corresponds to $\left.b^{2}\right|_{V_{1}^{\prime} \cup W_{1}^{\prime}}$. Therefore, by the above diagrams and a natural identification

$$
\mathrm{MCG}_{+}\left(M_{1}, V_{1} \operatorname{rel} D_{1}\right) \cong \mathrm{MCG}_{+}\left(M, V \operatorname{rel} V_{2}^{\prime} \cup W_{2}^{\prime}\right)
$$

it follows that $f_{2}$ can be written as a composition of a finite number of $\tilde{w}_{i}$ $(i \in\{1,2, \ldots, n\}), l_{j}^{ \pm 1}, m_{j}^{ \pm 1}(j \in\{1,2, \ldots, g-1\})$, and $b^{ \pm 2}$ up to isotopy preserving $V$. 

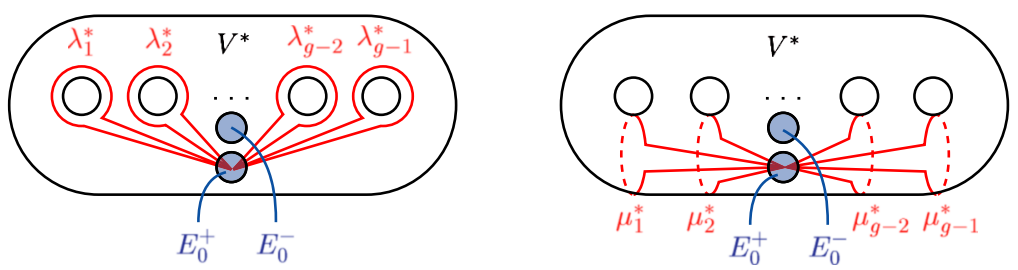

Figure 11

Suppose that $g=2$. Then instead of the first diagram in the previous argument, we have the following simpler diagram:

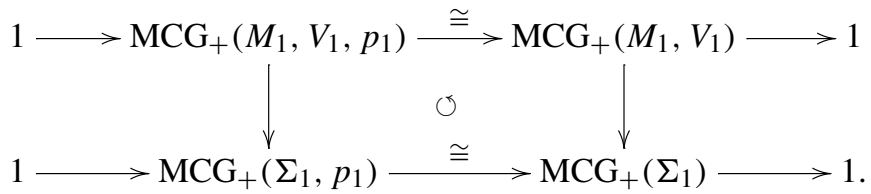

Hence, $f_{2}$ can be written as the composition of a finite number of $\tilde{w}_{i}$ ( $i \in$ $\{1,2, \ldots, n\})$ and $b^{ \pm 2}$ up to isotopy preserving $V$. This completes the proof.

In addition to the elements $\tilde{\omega}_{i}, \mu_{j}, \lambda_{j}, \beta$, and $\epsilon$, we define the elements $\lambda_{j}^{*}$ and $\mu_{j}^{*}$ of $\mathcal{G}(V, W ; \Sigma)$ for each $j \in\{1,2, \ldots, g-1\}$ as follows. Let $V^{*}$ be the handlebody $V$ cut off by the unique reducing disk $E_{0}$. Let $E_{0}^{+}$and $E_{0}^{-}$be disks in $\partial V^{*}$ coming from $E_{0}$. The elements $\lambda_{j}^{*}$ and $\mu_{j}^{*}$ for each $j \in\{1,2, \ldots, g-1\}$ are defined by pushing $E_{0}^{+}$along the arcs depicted in Figure 11. Each of these maps is realized by sliding a foot of the 1-handles $\operatorname{Nbd}\left(E_{0} ; V\right)$ and $\operatorname{Nbd}\left(E_{0}^{\prime} ; W\right)$ of $V$ and $W$, respectively, where $E_{0}^{\prime}$ is a disk in $W$ bounded by $\partial E_{0}$. We observe that, for any simple arcs $\gamma$ and $\gamma^{\prime}$ on $\partial V^{*}$ connecting $\partial E_{0}^{+}$and $\partial E_{0}^{-}$, there exists an element $\varphi$ of $\mathcal{G}(V, W ; \Sigma)$, which is a finite product of $\beta, \lambda_{j}^{*}$ and $\mu_{j}^{*}$ for $j \in\{1,2, \ldots, g-1\}$, such that $\varphi$ has a representative map sending $\gamma^{\prime}$ to $\gamma$.

Now let $\psi$ be any element of $\mathcal{G}(V, W ; \Sigma)$. Then $\psi([P])$ is also a vertex of the complex $\mathcal{H}_{E_{0}}$ by Proposition 2.3. If $\psi([P])=[P]$, then $\psi$ is a finite product of the elements $\tilde{\omega}_{i}, \mu_{j}, \lambda_{j}, \beta$, and $\epsilon$ by Lemma 3.2. Suppose that $\psi([P]) \neq[P]$. Then by Lemma 2.1 there exists a finite product, say $\varphi$, of $\beta, \lambda_{j}^{*}$, and $\mu_{j}^{*}$ such that $\varphi(\psi([P]))=[P]$. Thus, the composition $\varphi \circ \psi$ preserves the equivalence class of $P$, and consequently $\psi$ is a finite product of $\tilde{\omega}_{i}, \lambda_{j}, \mu_{j}, \lambda_{j}^{*}, \mu_{j}^{*}, \beta$, and $\epsilon$. We summarize this observation as follows.

TheOREM 3.3. Let $(V, W ; \Sigma)$ be the Heegaard splitting obtained from a genus$(g-1)$ splitting $\left(V_{1}, W_{1} ; \Sigma_{1}\right)$ for a 3-manifold and the genus-1 splitting $\left(V_{2}, W_{2} ; \Sigma_{2}\right)$ for $S^{2} \times S^{1}$, where $g \geq 2$. Suppose that there exists a unique reducing disk $E_{0}$ in $V$. If the Goeritz group of $\left(V_{1}, W_{1} ; \Sigma_{1}\right)$ is finitely generated, then the Goeritz group of $(V, W ; \Sigma)$ is also finitely generated. Moreover, under the given setting, the Goeritz group of $(V, W ; \Sigma)$ is generated by $\tilde{\omega}_{i}, \lambda_{j}, \mu_{j}, \lambda_{j}^{*}$, $\mu_{j}^{*}, \beta$, and $\epsilon$, where $i \in\{1,2, \ldots, m\}$ and $j \in\{1,2, \ldots, g-1\}$. 
By Proposition 2.6 and Theorems 3.1 and 3.3 we have the following:

Corollary 3.4. Let $\left(V_{1}, W_{1} ; \Sigma_{1}\right)$ be a genus- $(g-1)$ Heegaard splitting of Hempel distance at least 4 for a closed orientable 3-manifold $M_{1}$, where $g \geq 2$, and let $\left(V_{2}, W_{2} ; \Sigma_{2}\right)$ be the genus-1 Heegaard splitting for $S^{2} \times S^{1}$. If $(V, W ; \Sigma)$ is the splitting for $M_{1} \#\left(S^{2} \times S^{1}\right)$ obtained from $\left(V_{1}, W_{1} ; \Sigma_{1}\right)$ and $\left(V_{2}, W_{2} ; \Sigma_{2}\right)$, then the Goeritz group of the splitting $(V, W ; \Sigma)$ is finitely generated.

We note that Corollary 3.4 implies, in particular, that the Goeritz group of the genus-2 Heegaard splitting for $M_{1} \#\left(S^{2} \times S^{1}\right)$, where $M_{1}$ is $S^{3}$ or a lens space, is finitely generated, which is shown in [6] and [7].

ACKnOwledgments. Part of this work was carried out while the second-named author was visiting Università di Pisa as a JSPS Postdoctoral Fellow for Research Abroad. He is grateful to the university and its staff for the warm hospitality.

\section{References}

[1] E. Akbas, A presentation for the automorphisms of the 3-sphere that preserve a genus two Heegaard splitting, Pacific J. Math. 236 (2008), 201-222.

[2] J. S. Birman, Braids, links and mapping class groups, Ann. of Math. Stud., 82, Princeton Univ. Press, Princeton, NJ, 1974.

[3] S. Cho, Homeomorphisms of the 3-sphere that preserve a Heegaard splitting of genus two, Proc. Amer. Math. Soc. 136 (2008), 1113-1123.

[4] __ Genus two Goeritz groups of lens spaces, Pacific J. Math. 265 (2013), 1-16.

[5] S. Cho and Y. Koda, Primitive disk complexes for lens spaces, arXiv:1206.6243.

[6] - The genus two Goeritz, group of $S^{2} \times S^{1}$, Math. Res. Lett. 21 (2014), no. 3, 449-460.

[7] _ Disk complexes and genus two Heegaard splittings for nonprime 3manifolds, Int. Math. Res. Not. IMRN 12 (2015), 4344-4371.

[8] S. Cho, D. McCullough, and A. Seo, Arc distance equals level number, Proc. Amer. Math. Soc. 137 (2009), 2801-2807.

[9] B. Farb and D. Margalit, A primer on mapping class groups, Princeton Math. Ser., 49, Princeton University Press, Princeton, NJ, 2012.

[10] L. Goeritz, Die Abbildungen der Brezelfäche und der Vollbrezel vom Geschlecht 2, Abh. Math. Semin. Univ. Hambg. 9 (1933), 244-259.

[11] U. Hamenstädt and S. Hensel, The geometry of the handlebody groups I: distortion, J. Topol. Anal. 4 (2012), 71-97.

[12] W. Haken, Some results on surfaces in 3-manifolds, Studies in modern topology, pp. 39-98, Math. Assoc. Amer., Prentice-Hall, Englewood Cliffs, NJ, 1968.

[13] A. Hatcher, On triangulations of surfaces, Topology Appl. 40 (1991), 189-194.

[14] J. Hempel, 3-manifolds as viewed from the curve complex, Topology 40 (2001), 631657.

[15] E. Irmak and J. D. McCarthy, Injective simplicial maps of the arc complex, Turkish J. Math. 34 (2010), 339-354.

[16] J. Johnson, Mapping class groups of medium distance Heegaard splittings, Proc. Amer. Math. Soc. 138 (2010), 4529-4535.

[17] _ Mapping class groups of once-stabilized Heegaard splittings, arXiv:1108.5302. 
[18] M. Korkmaz and A. Papadopoulos, On the arc and curve complex of a surface, Math. Proc. Cambridge Philos. Soc. 148 (2010), 473-483.

[19] F. Lei, Haken spheres in the connected sum of two lens spaces, Math. Proc. Cambridge Philos. Soc. 138 (2005), 97-105.

[20] F. Lei and Y. Zhang, Haken spheres in genus 2 Heegaard splittings of nonprime 3manifolds, Topology Appl. 142 (2004), 101-111.

[21] D. McCullough, Virtually geometrically finite mapping class groups of 3-manifolds, J. Differential Geom. 33 (1991), 1-65.

[22] H. Namazi, Big Heegaard distance implies finite mapping class group, Topology Appl. 154 (2007), 2939-2949.

[23] M. Scharlemann and A. Thompson, Unknotting tunnels and Seifert surfaces, Proc. Lond. Math. Soc. (3) 87 (2003), 523-544.

[24] M. Scharlemann, Automorphisms of the 3-sphere that preserve a genus two Heegaard splitting, Bol. Soc. Mat. Mexicana (3) 10 (2004), 503-514.

[25] F. Waldhausen, Heegaard-Zerlegungen der 3-Sphäre, Topology 7 (1968), 195-203.

S. Cho

Department of Mathematics Education

Hanyang University

Seoul 133-791

Korea

scho@hanyang.ac.kr
Y. Koda

Department of Mathematics

Hiroshima University

1-3-1 Kagamiyama

Higashi-Hiroshima, 739-8526

Japan

ykoda@hiroshima-u.ac.jp

A. Seo

Department of Mathematics

Education

Korea University

Seoul 136-701

Korea

arimseo@korea.ac.kr 